\title{
Évaluer l'utilité dans le contexte des technologies émergentes pour identifier des besoins latents : éléments issus d'une analyse des interactions en situation d'usage
}

Evaluating usefulness in the context of emerging technologies to identify latent needs: elements from an analysis of the interactions in in-use

Émilie Loup-Escande et Jean-Marie Burkhardt

\section{OpenEdition}

Journals

Édition électronique

URL : http://journals.openedition.org/activites/4554

DOI : 10.4000/activites.4554

ISSN : $1765-2723$

Éditeur

ARPACT - Association Recherches et Pratiques sur les ACTivités

Référence électronique

Émilie Loup-Escande et Jean-Marie Burkhardt, «Évaluer l'utilité dans le contexte des technologies émergentes pour identifier des besoins latents : éléments issus d'une analyse des interactions en situation d'usage ", Activités [En ligne], 16-2 | 2019, mis en ligne le 15 octobre 2019, consulté le 17 octobre 2019. URL : http://journals.openedition.org/activites/4554; DOI : 10.4000/activites.4554

Ce document a été généré automatiquement le 17 octobre 2019.

\section{(i) $9 \Theta$}

Activités est mis à disposition selon les termes de la licence Creative Commons Attribution - Pas d'Utilisation Commerciale - Pas de Modification 4.0 International. 


\section{Évaluer l'utilité dans le contexte des technologies émergentes pour identifier des besoins latents : éléments issus d'une analyse des interactions en situation d'usage}

Evaluating usefulness in the context of emerging technologies to identify latent needs: elements from an analysis of the interactions in in-use

Émilie Loup-Escande et Jean-Marie Burkhardt

\section{NOTE DE L'ÉDITEUR}

Article soumis le 31 mai 2018, accepté le 24 janvier 2019

Nous remercions Laval Agglomération et le Conseil Général de la Mayenne pour leur soutien, le partenaire industriel qui a contribué à ces études de terrain, ainsi que l'Institut Français du Textile et de l'Habillement, porteur du projet «3D Child».

\section{Introduction}

1 L'utilité, considérée comme un critère majeur en ergonomie (voir Haradji, \& Faveaux, 2006 ; Burkhardt, \& Sperandio, 2004) désigne très généralement le degré d'adéquation entre les buts poursuivis par les utilisateurs et les buts rendus possibles par l'utilisation de l'artefact. L'utilité est cependant une propriété construite, évolutive (Loup-Escande, Burkhardt, \& Richir, 2013) et "située ». Il s'agit d'une propriété construite dans la mesure où elle ne préexiste pas à l'objet conçu et émerge de l'interaction entre les acteurs concepteurs et/ou utilisateurs, sur la base d'objets intermédiaires et de 
représentations diverses mobilisées autour de la conception. L'utilité est évolutive en ce sens qu'elle n'est ni complète ni définitive à chaque instant du processus de conception, mais change au cours du temps, en fonction de la façon dont changent les utilisateurs, l'outil et l'environnement. Cette propriété dynamique fondamentale de l'utilité est cruciale en particulier dans le cadre des technologies émergentes. Ces dernières se caractérisent en effet par un caractère novateur, des usages peu clairs et peu différenciés, plusieurs limites qui en ralentissent l'application massive, et une promesse de transformation du contexte économique et social, dans lequel elle sera introduite (Anastassova, 2006; Kjeldskov, 2003). ${ }^{1}$ Il en découle des difficultés importantes pour la conception de ces technologies, notamment pour anticiper et apprécier l'utilité d'un futur artefact avant de figer les fonctions, usages et caractéristiques dans la solution finale. Enfin, l'utilité est « située » dans le sens où elle dépend grandement du contexte (existence ou non d'alternatives que l'utilisateur est à même de mobiliser pour atteindre les mêmes objectifs, autres outils existants ou habituellement utilisés, caractéristiques spécifiques à l'environnement d'utilisation, interdépendance avec d'autres activités, inscription dans le temps, etc.; cf. Burkhardt, \& Sperandio, 2004), mais aussi de la capacité des utilisateurs à formuler voire à imaginer des buts pertinents en regard d'une nouvelle technologie disponible.

2 L'utilité a été aussi définie comme l'adéquation de l'artefact avec les besoins de l'utilisateur (Bastien, \& Tricot, 2008 ; Haradji, \& Faveaux, 2006), c'est-à-dire la propriété de l'artefact qui résulte de la prise en compte effective ou non des besoins des utilisateurs. Dans ce cadre, la notion de besoin renvoie d'un côté à la fonction ou au service attendu ou souhaité par l'utilisateur, et de l'autre côté à la force de cette attente, voire son degré de pertinence. Pour concevoir un artefact utile, l'enjeu réside dans l'identification de besoins des utilisateurs, puis dans leur traduction efficace au niveau de l'artefact issu du processus de conception (pour une discussion plus large de la notion de besoin dans la conception en lien avec l'utilité, voir Loup-Escande, Burkhardt, \& Richir, 2013).

3 Le présent article s'intéresse à l'expression des besoins latents dans un contexte de technologies fortement innovantes dans une approche rétrospective de la conception (par opposition à une approche prospective), c'est-à-dire une approche s'appuyant sur la mise en situation d'utilisateurs dans un contexte écologique et en forte interaction collaborative avec un représentant de l'équipe de conception. Les besoins latents sont des besoins que les utilisateurs n'ont pas encore imaginés (Robertson, 2001). Ces besoins sont, à un moment donné, non conscientisés (Murray, 1938), et de fait non exprimables par les utilisateurs. Ils se caractérisent également par leur nature non encore avérée (Sperandio, 2001). Ce type de besoins latents est un enjeu important pour les technologies émergentes, encore au stade du développement en laboratoire, et donc en devenir, dans la mesure où elles sont en recherche de leurs applications. Or, anticiper la technologie et les applications qui vont « marcher » constitue l'un des défis actuels de l'innovation intensive (Durand, Jumel, \& Pons, 2003).

L'objectif de cet article est d'étudier dans quelle mesure l'évaluation de l'utilité d'une technologie émergente en contexte écologique favorise la génération de besoins latents lors d'interactions en contexte écologique entre un concepteur et des utilisateurs, sur la base de l'utilisation d'une version hautement fidèle d'un logiciel en cours de développement. Les interactions ont en effet lieu au sein des entreprises utilisatrices et dans le cadre de situations de formation et de travail propres à leur domaine d'activité. 
L'étude porte sur un projet de développement d'une application de type desktop-VR ${ }^{2}$ appelée "Appli-Viz'3D » visant à assister l'activité de conception de produits de puériculture pour différents profils d'utilisateurs (ingénieurs, designers et marketeurs). Ce logiciel permet à ces utilisateurs de mettre en scène des prototypes virtuels (par ex. table à langer) et des avatars dans des environnements virtuels réalistes (par ex. une chambre d'enfants) afin de les aider à évaluer rapidement les concepts (Loup-Escande, Burkhardt, Christofol, \& Richir, 2010). Cette étude s'inscrit dans le cadre de l'évaluation ergonomique de l'utilité, vue comme un déterminant -parmi d'autres - de l'acceptation du dispositif sur le long terme.

5 La suite de l'article est organisée comme suit. La partie suivante discute de l'évaluation orientée vers l'utilité comme facilitatrice de la production de spécifications candidates. La conception pour (ou de) l'utilité est une approche adoptant une perspective centrée sur le critère ergonomique de l'« utilité » tout en s'inscrivant dans la continuité des modèles et des approches ergonomiques de la conception pluridisciplinaire, participative et continue au travers de l'usage (cf. Loup-Escande, 2011). Nous présentons ensuite l'étude menée à partir de deux situations de terrain (une situation de formation et une situation d'utilisation) où nous montrons notamment que les utilisateurs, avec l'aide d'un concepteur-animateur, deviennent capables de verbaliser des besoins latents lorsqu'ils sont mis en situation écologique d'utilisation de la technologie émergente en cours de conception. Nous discutons ensuite nos résultats en regard notamment de la pertinence de ces interactions situées entre concepteur et utilisateurs non seulement pour l'apprentissage mutuel sous-jacent nécessaire à la finalisation de la conception de la technologie émergente, mais aussi pour former et accompagner son introduction dans l'organisation. En conclusion, nous reprenons les apports et les limites de l'approche rétrospective dans le contexte de la conception de l'utilité des technologies émergentes, avant d'évoquer la nécessité de mener également des recherches s'inscrivant dans l'approche prospective.

\section{De la mesure et l'évaluation de l'utilité à la production de spécifications candidates: caractéristiques d'une approche rétrospective}

\subsection{La conception pour l'utilité : apports réciproques entre l'approche prospective et l'approche rétrospective}

6 Nous distinguons deux directions d'approches principales dans la conception: prospective et rétrospective (Figure 1). Ces deux approches coexistent et alimentent complémentairement les spécifications candidates résultant de l'univers prospectif et de l'univers rétrospectif de l'utilité (Loup-Escande, Burkhardt, \& Richir, 2013). En effet, la conception implique une dialectique continuelle entre les deux univers depuis l'amont, où tant l'utilité que la forme et le détail de l'artefact restent flous et hypothétiques, jusqu'à l'aval où la forme finale est éprouvée et continue à évoluer dans l'usage. L'univers prospectif regroupe l'ensemble des particularités, besoins ou solutions pensables (mais pas forcément souhaitables), tandis que l'univers rétrospectif est assimilable à l'ensemble des expériences pertinentes en termes d'utilité pour informer la conception de l'artefact. 
7 Les spécifications candidates extraites des deux univers précités concernent la destination de l'artefact (c'est-à-dire, les fonctionnalités et des propriétés inscrites dans le système pouvant prendre des formes différentes en fonction du moment dans la conception), mais aussi la plus-value pour les utilisateurs (c'est-à-dire un avantage significatif du système pour l'utilisateur, avantage étant relatif aux objectifs de l'utilisateur, aux alternatives que l'utilisateur est à même de mobiliser pour atteindre les mêmes objectifs, aux outils existants ou habituellement utilisés, à l'environnement d'utilisation, aux dépendances avec les autres activités et au temps). En outre, il est évidemment hautement souhaitable que l'utilité (au sens de la destination) de l'objet conçu corresponde à une utilité (au sens de la plus-value) « réelle », tangible pour celles ou ceux qui vont l'utiliser.

8 Pour aboutir aux spécifications candidates, deux approches de la conception pour l'utilité non mutuellement exclusives coexistent: l'approche prospective (par ex. Brangier, \& Robert, 2014) et l'approche rétrospective, c'est-à-dire fondée sur l'évaluation (par ex. Anastassova, Mégard, \& Burkhardt, 2007). L'approche prospective de l'utilité correspond aux possibles envisagés par chaque acteur de la conception, relevant de la prédiction, de la projection ou encore de l'imagination des fonctions et de la forme de l'outil voire des futurs usages. L'approche rétrospective de l'utilité renvoie à une (re)construction des fonctions et des usages, sur la base de l'état actuel ou d'états précédents de l'outil, en tenant compte des propriétés de l'environnement d'utilisation et de l'état des connaissances de l'utilisateur (Loup-Escande, Burkhardt, \& Richir, 2013).

En d'autres termes, l'approche prospective fait référence aux actions des acteurs de la conception (c'est-à-dire, la prédiction, la projection et l'imagination) qui permettent de passer d'un univers prospectif (c'est-à-dire, besoins et solutions possibles envisagés) à des spécifications candidates pour la conception de la technologie. L'approche rétrospective concerne les actions (c'est-à-dire, la (re)construction des fonctions et des usages) qui permettent de passer d'un univers rétrospectif (c'est-à-dire, expériences antérieures des utilisateurs avec des états intermédiaires de l'outil) aux spécifications candidates. 
Figure 1 : Relations entre univers prospectif, univers rétrospectif, spécifications candidates, utilité potentielle et utilité réelle.

Figure 1: Relations between prospective and retrospective worlds, candidate specifications, potential and effective usefulness

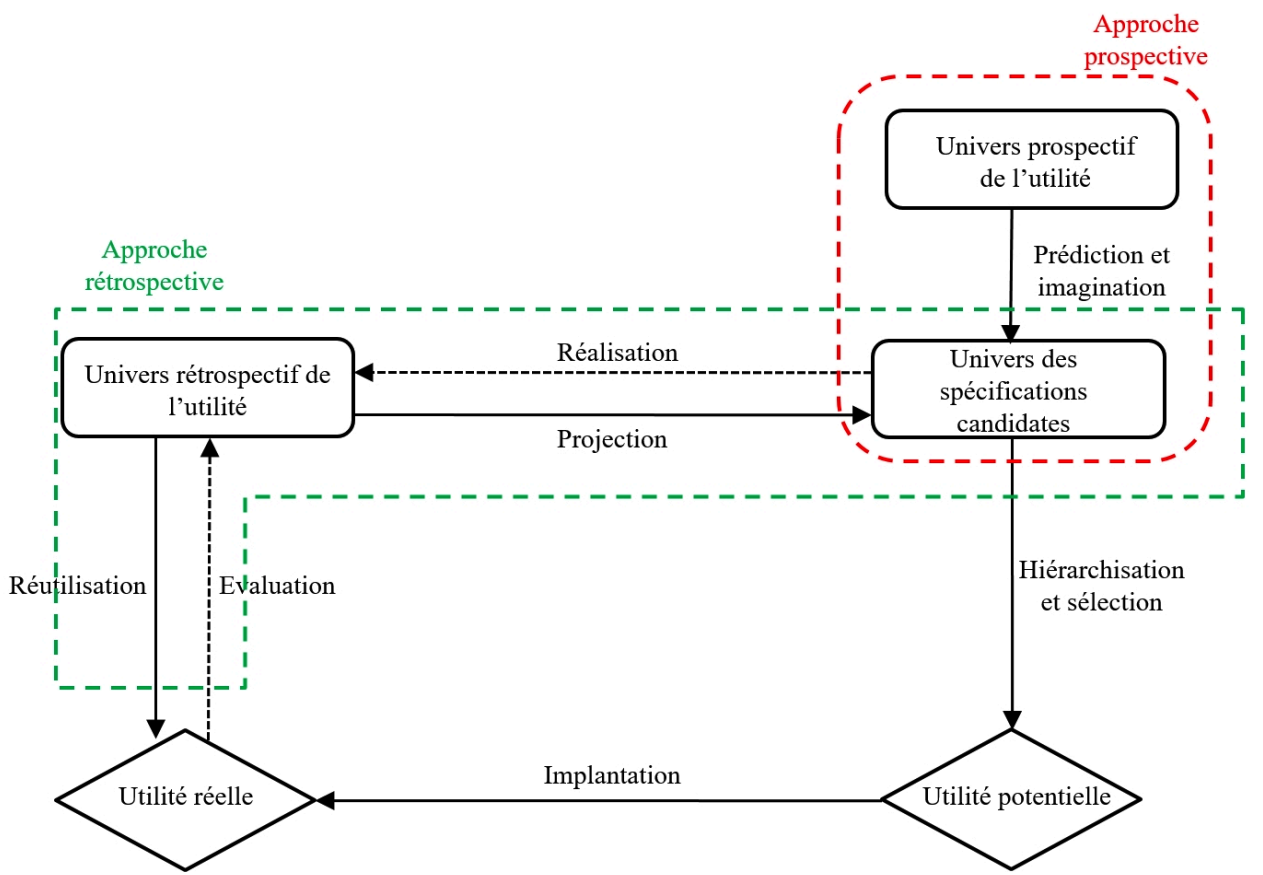

\subsection{L'évaluation de l'utilité : une collaboration entre concepteurs et utilisateurs} mesurer, d'estimer, de déterminer la valeur ou encore de juger. En ergonomie, et pour le cadre particulier de la conception des technologies, l'évaluation peut répondre à plusieurs objectifs (par ex. concevoir, sélectionner, accompagner l'introduction de la technologie dans une situation de travail ou d'apprentissage, etc.) et concerner des cibles de nature et de complexité différentes (par ex. efficacité d'une métaphore d'interaction, performance d'un logiciel, etc.). La tendance actuelle articule deux formes d'évaluation complémentaires dans les projets: l'évaluation formative et l'évaluation sommative. L'évaluation sommative vise à mesurer la qualité d'un système et/ou quantifier la performance des participants afin de les situer soit en référence à un standard attendu, soit par comparaison avec des alternatives en compétition. Ce type d'évaluation repose essentiellement sur une approche quantitative (au travers de divers outils de recueil tels que le questionnaire, l'enregistrement de données physiologiques, etc.). L'évaluation formative vise à produire des données qualitatives (par ex. matériel verbal contenu dans le discours de sujets, réponses à des questions ouvertes, etc.) ou mixtes quantitative/qualitative sur l'activité observée et les processus mis en œuvre par les utilisateurs dans leurs interactions avec un système, sur les difficultés rencontrées et les facteurs explicatifs associés, sur l'identification de besoins non pourvus, voire sur des idées de solutions aux problèmes rencontrés.

L'ergonomie dispose de critères et de méthodes variés selon que l'on évalue l'utilité au sens de la destination (ce que l'artefact permet de faire, les buts qu'il permet d'atteindre) ou l'utilité au sens de la valeur (en quoi l'atteinte de ces buts au moyen de 
l'artefact proposé constitue un avantage réel et significatif pour l'utilisateur dans son contexte). Typiquement, l'évaluation des choix relatifs aux fonctions et à la spécification détaillée de leur mise en œuvre implique l'élaboration de critères de mesure de la pertinence des « besoins ». Ces critères, issus éventuellement des analyses amont - par ex. l'analyse du travail - sont potentiellement enrichis par l'identification de nouveaux besoins au fur et à mesure du projet, au travers de méthodes de mises en situation (par ex. tests utilisateurs, simulations) ou au travers de méthodes analytiques étudiant les spécifications implémentées dans le système (par ex. Blandford, Green, Furniss, \& Makri, 2008). Concernant l'utilité au sens de la valeur, il convient de distinguer d'un côté l'évaluation de l'avantage relatif de l'artefact par rapport à l'existant et au contexte, en regard des multiples dimensions impliquées (coût, efficacité, utilisabilité de l'interface homme-machine (Dix, Finlay, Abowd, \& Beale, 2004 ; Nielsen, 1993), et de l'autre côté la mesure de l'acceptabilité sociale des technologies visant à identifier les représentations défavorables, les craintes et les facteurs socio-cognitifs qui impactent l'adoption de ces dispositifs basée sur la représentation subjective de l'utilité possible de cette technologie et non son expérience effective (Terrade, Pasquier, Reerink-Boulanger, Guingouain, \& Somat, 2009). Dans le premier cas, l'utilité peut se mesurer selon des critères objectifs et en situation écologique. Dans le second cas, elle s'évalue selon des modalités subjectives comme l'utilité ou l'utilisabilité perçues (Davis, 1989 ; Brooke, 1996).

L'évaluation de l'utilité d'un artefact implique une collaboration entre concepteurs et utilisateurs et peut prendre place à des instants différents de la vie du processus de conception (c.-à-d., évaluation d'un prototype hautement fidèle, évaluation du produit final avant utilisation en entreprise, évaluation en entreprise lorsque le système est en cours d'utilisation) afin d'en mesurer la «valeur» associée pour l'utilisateur ou le groupe d'utilisateurs, y compris même avant le lancement d'un projet de conception. Dans ce dernier cas, les ergonomes, comme les ingénieurs, analysent l'existant afin de faire des hypothèses sur un besoin ou un manque potentiel. Dans une démarche ergonomique, l'évaluation à ce stade préliminaire de la conception implique parfois uniquement des concepteurs (par ex. via des inspections expertes), ou bien nécessite d'intégrer des utilisateurs notamment lorsqu'il s'agit de les questionner sur leurs attentes voire de les observer dans un contexte donné pour identifier les manques auxquels le système envisagé pourrait pallier. Toutefois, la mesure de l'utilité ne devrait pas se restreindre à cette étape préliminaire, mais s'étendre aux phases ultérieures du processus de conception. La conception participative repose précisément sur l'idée selon laquelle l'évolution de l'utilité d'un système nécessite une analyse audelà de la phase préalable de recueil de besoins, c'est-à-dire durant la réalisation technique de l'artefact, et en aval de la conception, et doit impliquer les utilisateurs. Cette conception participative implique un apprentissage mutuel entre concepteurs et utilisateurs grâce auquel l'outil technologique et l'activité des utilisateurs se développent conjointement (Béguin, 2003). Créer des conditions optimales pour favoriser cet apprentissage reste aujourd'hui un enjeu. De plus, s'il n'est pas rare de confronter les utilisateurs à des prototypes dans des situations expérimentales simplifiées (par ex. Loup-Escande, Dominjon, Perret, Erhel, Jamet, Michinov, Andriot, Gravez, \& Ragot, 2013), un risque réside dans l'impossibilité de recueillir les besoins latents associés à l'usage dans l'environnement réel d'utilisation. Aussi, confronter les utilisateurs dans des contextes réels d'usage permettrait de contextualiser l'expression de besoins, d'identifier des besoins latents situés et pertinents en regard de la situation 
et ainsi d'améliorer l'utilité de l'artefact. Les études en ergonomie qui ont cherché à évaluer la pertinence des fonctionnalités d'un dispositif (c'est-à-dire, l'utilité au sens de la destination) dans le cadre de situations écologiques reposent essentiellement sur la base de l'activité outillée des utilisateurs (par ex. Bourmaud, 2008; Rabardel, 1995), sans étudier finement la contribution de cette évaluation et des interactions situées entre les concepteurs et les utilisateurs, à l'évolution de l'utilité de l'artefact en cours de développement.

\subsection{L'évaluation de l'existant pour alimenter l'univers rétrospectif de l'utilité}

\subsubsection{L'inspection des interfaces par les concepteurs pour enrichir la destination des artefacts}

13 Un premier type d'analyse pratiquée dans le domaine des technologies est l'identification des défaillances par les concepteurs eux-mêmes (Nogier, 2005). Ces méthodes sont caractérisées par l'absence de recours aux utilisateurs finaux. Elles reposent sur des normes, des guides de styles et des recommandations adaptés (par ex. Bastien, \& Scapin, 1993; Bach, \& Scapin, 2005) voire sur des heuristiques (par ex. Stanney, Mollaghasemi, Reeves, Breaux, \& Graeber, 2003) qui permettent de réaliser des inspections expertes (par ex. Schmettow, Bach, \& Scapin, 2014) ou des inspections cognitives (par ex. Tromp, Steed, \& Wilson, 2003). Ces critères peuvent être utilisés afin de découvrir et de corriger des défauts de conception, avant de réaliser des tests utilisateurs (Bastien, \& Scapin, 1993). Il résulte d'une telle évaluation, une suite de préconisations fondées sur la représentation d'un tiers qui n'est pas l'utilisateur final. Ce tiers peut être un ergonome, un designer ou un ingénieur ayant a priori une meilleure connaissance des technologies à mettre en œuvre que les utilisateurs réels. Par conséquent, une limite de ce type d'évaluation réside dans le fait de réaliser des évaluations hors situation réelle de travail et sans utilisateur, ce qui conduit le concepteur à se faire une idée fausse ou partielle de la tâche et à se construire sa propre représentation qui n'est pas celle des attentes et besoins souhaités par les utilisateurs (Darses, \& Wolff, 2006). L'évaluation pourra alors conclure que les fonctionnalités sont pertinentes sans qu'elles apportent un bénéfice réel à l'utilisateur.

\subsubsection{La confrontation des utilisateurs à l'artefact pour faire correspondre la destination du logiciel à une plus-value « réelle »}

$14 \mathrm{Au}$ travers de la réalisation et de la mise en situation, la mise à l'épreuve de l'utilité engendre une réflexion sur le besoin voire le pousse à évoluer. Cette mise en situation peut passer par plusieurs méthodes comme l'évaluation d'objets intermédiaires (par ex. les prototypes, les maquettes), les scénarios et les cas d'utilisation (Carroll, 2000). L'évaluation de prototypes peut s'appuyer sur une activité de simulation à des fins de reconstitution d'une situation de travail ou d'utilisation, dans le but d'induire des comportements susceptibles d'apparaitre dans la situation future et de s'en saisir pour mettre en discussion le projet et le faire évoluer. D'une part, il s'agit d'une mise en situation grandeur nature qui va notamment permettre aux utilisateurs de confronter les «invariants» qui guident leur activité aux circonstances associées au projet en développement (Bobillier Chaumon, Rouat, Laneyrie, \& Cuvillier, 2018). L'observation des impacts effectifs de l'artefact sur les activités des utilisateurs permet d'identifier les 
conditions qui permettront aux utilisateurs de tirer un réel bénéfice de leur utilisation (Burkhardt, \& Lubart, 2010). D'autre part, les prototypes constituent des supports à la compréhension des concepts technologiques flous pour les participants qui n'ont pas une expertise technologique suffisante comme les futurs utilisateurs. Ils aident les utilisateurs à développer les conceptualisations dont ils ont besoin pour en parler avec les concepteurs (Anastassova, Burkhardt, \& Mégard, 2007). Enfin, les prototypes sont également un moyen pour projeter les utilisateurs dans un contexte d'usage futur intégrant le logiciel en cours de conception. Les utilisateurs sont alors en capacité de proposer des fonctionnalités de l'application future jusque-là latentes et nonconscientes, et contribuent de fait à enrichir l'utilité-destination en intégrant ces nouveaux besoins au cours de la conception (Newell, Carmichael, Morgan, \& Dickinson, 2006). Comme souligné par Bobillier Chaumon, Rouat, Laneyrie et Cuvillier (2018), le recours à la simulation en conception peut ainsi impliquer plusieurs dimensions complémentaires pertinentes pour la conception dans le cadre des technologies émergentes, notamment sous-tendre une orientation rétrospective (fondée sur l'expérience passée) et prospective (tournée vers une activité à venir) ainsi qu'un soutien à la réflexivité du collectif de participants.

Malgré les apports précédemment évoqués, la conception par prototypage présente des limites en particulier s'ils sont très/trop éloignés de la situation future en termes de matériel, de contenu et de fonctionnalités. Ainsi, les résultats de l'évaluation réalisée en termes de "preuve de concept " avec un prototype basse fidélité peuvent être peu prédictifs et n'apporter aucune réponse quant aux spécificités du produit final. Les utilisateurs peuvent par ailleurs se projeter dans un contexte erroné et construire une représentation de l'outil intermédiaire non représentative de la situation et de l'outil futur. Ces éléments incitent à la réalisation d'évaluations complémentaires mobilisant des prototypes hautement fidèles (c'est-à-dire, quasi finalisés en termes de fonctionnalités et de propriétés, et dont les développements informatiques ultérieurs restent mineurs), voire sur l'outil final. Ces évaluations doivent s'inscrire dans le cadre d'une démarche de conception centrée-utilisateur et itérative.

\subsection{Des séances collectives pour favoriser l'expression de spécifications candidates}

Les séances collectives de conception et d'évaluation de l'artefact incluant les concepteurs et/ou les utilisateurs finaux favorisent l'expression de besoins et d'alternatives de solutions. Ces séances collectives mobilisent des méthodes telles que les Focus Group (par ex. Bruseberg, \& McDonagh-Philip, 2002) qui favorisent la production de concepts innovants au sens de besoins non couverts ou latents, grâce à une atmosphère créative que les auteurs expliquent par :

- Un environnement informel et amical qui accroît la confiance entre les utilisateurs et les concepteurs;

- L'opportunité de partager des expériences, des opinions et des idées qui peut conduire à une plus profonde immersion dans le problème de conception.

17 Ces situations collectives permettraient ainsi de passer de l'univers rétrospectif, contenu dans des systèmes concurrents ou dans un artefact en cours de conception, aux spécifications candidates. Ce processus qualifié de « projection » dans notre modèle (Figure 1) a été analysé dans plusieurs études, notamment au travers de l'analyse des 
données verbales produites lors de réunions de conception (Darses, Détienne, Falzon, \& Visser, 2001), d'évaluation (D'Astous, Détienne, Visser, \& Robillard, 2004) et de réunions de validation (Karsenty, 1991). Ces études montrent que - quel que soit l'objectif des séances collectives de travail (conception, évaluation, validation) - des activités cognitives d'évaluation et de propositions de solutions sont mises en œuvre. Du point de vue de la conception centrée sur l'utilité, cela montre qu'il y a un passage continuel entre l'univers rétrospectif et la production de spécifications candidates, en particulier dans le cadre des réunions qui s'appuient sur un artefact. De par leur objectif qui est d'analyser l'activité collective de conception déployée par des concepteurs, les deux premières études n'incluent pas les utilisateurs de l'artefact, contrairement à la dernière. Il serait intéressant d'analyser des séances collectives d'évaluation incluant des utilisateurs en vue d'améliorer la prise en compte de l'utilité dans la conception.

En résumé, ce bilan de la littérature suggère que, dans le contexte de la conception de l'utilité d'un artefact innovant, l'évaluation d'un artefact existant par plusieurs acteurs (concepteurs et utilisateurs) lors de séances collectives pourrait constituer une approche originale et pertinente permettant de faire correspondre l'utilité-destination à une plus-value réelle en favorisant l'expression de besoins jusqu'alors non conscientisés par les utilisateurs. Toutefois, il n'existe pas, à notre connaissance, d'étude traitant explicitement de l'approche rétrospective de la conception de l'utilité dans un cadre réellement écologique (c'est-à-dire, dans le contexte réel d'utilisation) et collectif (c'est-à-dire, incluant des concepteurs et plusieurs profils d'utilisateurs réels). La recherche empirique proposée ci-après intègre ces spécificités.

\section{Exemple du logiciel Appli-Viz'3D chez un fabricant de mobilier : une recherche menée sur un terrain réel}

\subsection{Objectifs}

Deux objectifs scientifiques guident ce travail. Le premier est de présenter une démarche d'évaluation d'une technologie émergente en contexte écologique pour optimiser son utilité et en faciliter l'introduction au sein de l'entreprise. Pour ce faire, nous avons mis en place une situation de formation et une situation de travail adaptée pour laquelle le logiciel Appli-Viz'3D était particulièrement pertinent (la revue de projet d'un concept de meuble d'enfant). Le second objectif est de montrer en quoi les interactions entre les participants à de telles situations d'évaluation contribuent à la production de besoins latents, et de fait, à la conception de l'utilité du logiciel. Pour cela, nous avons réalisé une analyse cognitive des productions verbales entre les utilisateurs et le concepteur impliqués dans les situations précitées.

Comme exposée précédemment, l'approche rétrospective est particulièrement pertinente dans le cadre de situations de travail (par ex. Darses et al., 2001; D'Astous et al., 2004; Karsenty, 1991) et dans un contexte de formation en favorisant un apprentissage mutuel entre concepteurs et utilisateurs (Béguin, 2003). En effet, ces situations réunissent les conditions optimales pour étudier de façon approfondie le processus rétrospectif dans son ensemble fondé sur l'évaluation de l'existant en vue de générer des spécifications candidates visant à mesurer voire faire correspondre la destination de l'artefact à une plus-value réelle pour l'utilisateur. 
Deux situations d'évaluation ont été sélectionnées: la première est une situation de formation dans laquelle le logiciel était objet et support de la formation, assurée par le concepteur-stagiaire. La seconde étude est une situation de travail, en particulier une revue de projet dans laquelle la technologie émergente était un support de présentation des concepts de produits. En effet, la fabrication de meubles implique une phase préalable au lancement de la production alternant des étapes de « design » et de tests de prototypes virtuels puis physiques (Ulrich, \& Eppinger, 2003). Dans cette phase amont, des revues de projet régulières sont organisées. Il s'agit de séances durant lesquelles des ingénieurs, des designers et des marketeurs présentent, échangent voire valident un nouveau concept de produit (par ex. une table à langer).

\subsection{Méthode}

\subsubsection{Contexte de l'étude : le projet Appli-Viz'3D}

La présente recherche s'inscrit dans le cadre d'un projet nommé «3D Child » visant à concevoir le logiciel Appli-Viz'3D. Le logiciel utilisé dans le cadre de l'étude est un prototype hautement fidèle par rapport au logiciel final (Figure 2). Ce projet, porté par l'Institut Français du Textile et de l'Habillement, mobilisait des concepteurs (ingénieurs, designers et ergonome) des Arts et Métiers ParisTech et des utilisateurs (ingénieurs, designers et maketeurs) provenant d'une entreprise de 1050 salariés spécialisée dans la fabrication de mobilier, notamment pour enfants.

L'ergonome avait un double statut de chercheure et de cheffe de projet. En tant que doctorante en ergonomie, elle choisissait les situations à étudier, concevait les protocoles et outils de recueil, collectait et analysait les données pertinentes pour ses recherches. En tant que coordinatrice du projet pour les Arts et Métiers ParisTech, elle avait la possibilité de mettre en place des situations particulières telles que celles décrites dans cette publication.

Figure 2 : Capture d'écran d'une chambre d'enfant virtuelle dans l'application Appli-Viz'3D. Figure 2: Screenshot of a virtual child's room in the Appli-Viz'3D application

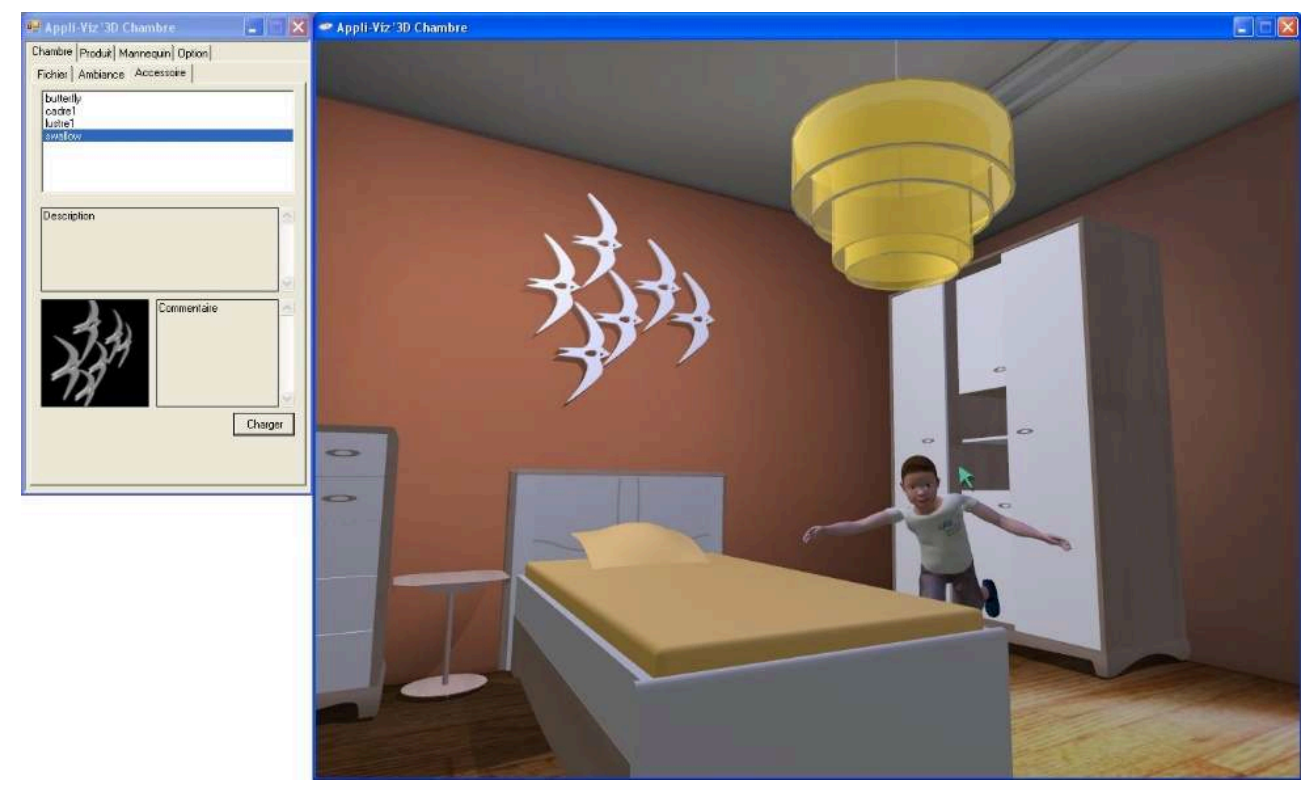


Ce projet s'appuie sur une démarche de Conception Centrée-Utilisateur (ISO 9241-210) au travers de quatre étapes. La première étape a consisté à comprendre et à spécifier le contexte d'utilisation au travers de l'analyse des caractéristiques des utilisateurs potentiels, d'une formalisation des tâches que l'utilisateur devra réaliser avec le logiciel et d'une analyse de l'environnement dans lequel il sera intégré. La deuxième étape a consisté à spécifier les besoins et les exigences de chaque partie prenante, en particulier des utilisateurs. La troisième étape a concerné la production de solutions de conception (par ex. maquettes ou prototypes) sur la base des connaissances techniques d'une part, des connaissances sur les utilisateurs et le contexte d'utilisation d'autre part. Enfin, la dernière étape concernait l'évaluation des solutions conçues par rapport aux contextes d'utilisation, aux caractéristiques et aux besoins des utilisateurs. L'étude présentée ici prend place dans la quatrième étape et en fin de projet quelques mois avant le déploiement dans l'entreprise. Les deux situations d'utilisation d'Appli-Viz'3D (la formation et la revue de projet) ont été intentionnellement construites par l'ergonome, dans une perspective d'évaluation de l'utilité en vue de répondre aux objectifs de recherche précités.

\subsubsection{Participants}

Un concepteur du logiciel et sept utilisateurs de l'entreprise de fabrication de meubles avec trois profils différents ont participé aux études. La situation de formation a impliqué le concepteur et quatre utilisateurs (1 ingénieur, 2 designers et 1 marketeur), tandis que la revue de projet mobilisait le concepteur et cinq utilisateurs (1 ingénieur, 2 designers ${ }^{3}$ et 2 marketeurs). Le concepteur était un élève-ingénieur en réalité virtuelle effectuant un stage de 6 mois au sein de l'entreprise. Cette posture de concepteur-stagiaire lui permettait non seulement d'accompagner les utilisateurs quant à l'intégration d'Appli-Viz'3D dans leur activité par la formation et l'accompagnement lors de revues de projet, mais aussi de finaliser le logiciel en fonction des retours des utilisateurs. Les sept utilisateurs, recrutés pour participer à ces séances sur la base du volontariat, avaient en moyenne 39 ans (E.T. $=10 ;$ Min $=28$; Max $=56)$ et 18 années d'expérience (E.T. $=11 ; \operatorname{Min}=5 ; \operatorname{Max}=35)$. Les designers se concentrent sur les attributs stylistiques (par ex. formes, couleurs, textures, aspects des matériaux) et les attributs d'usages (par ex. scénarios d'utilisation) du meuble. Les ingénieurs définissent et valident une gamme d'assemblage en tenant compte des liaisons cinématiques et s'assurent du respect des normes. Les marketeurs ont en charge la commercialisation du mobilier conçu. En ce qui concerne leurs habitudes, si les marketeurs n'utilisaient pas d'environnements de simulation, les ingénieurs étaient formés à des logiciels de simulation de type CATIA, les designers manipulaient régulièrement des logiciels d'infographie tels que 3 DS Max. Parmi les sept participants, deux ayant participé à la revue de projet étaient impliqués dans le projet depuis le début et avaient suivi l'évolution du logiciel.

\subsubsection{Procédure et consigne}

Lors de la formation et de la revue de projet, les utilisateurs avaient la même consigne : ils devaient configurer une chambre d'enfant « virtuelle » et y intégrer un concept de produit qu'ils avaient préalablement conçu afin de l'évaluer dans son environnement d'utilisation. Les situations investiguées et la consigne associée ont été élaborées à partir de données recueillies lors d'entretiens exploratoires et d'observations ouvertes 
des designers et des ingénieurs : les entretiens avaient notamment permis d'identifier la pertinence du logiciel pour les revues de projet, les observations avaient permis de formaliser une revue de projet sans l'outil et d'élaborer la consigne proposée dans les situations précitées.

Les utilisateurs qui manipulaient le logiciel ont eu pour consigne de verbaliser ce qu'ils souhaitaient faire et comment ils devaient le faire. Ils pouvaient aussi dialoguer avec les autres utilisateurs et/ou avec le concepteur. La seule différence entre les deux situations était que le concepteur avait également proposé aux utilisateurs de ne pas hésiter à poser des questions dans le cadre de la formation. En d'autres termes, le concepteur était présent pour guider les utilisateurs qui découvraient le logiciel en situation de formation, alors qu'il répondait aux interrogations ou aux incompréhensions ponctuelles des utilisateurs en situation de revue de projet.

Les deux situations s'appuyaient volontairement sur la même version du logiciel, version relativement complète en comparaison avec la version finale d'Appli-Viz'3D. Le scénario d'utilisation impliquait les mêmes fonctionnalités du fait de la consigne identique.

\subsubsection{Données recueillies}

Nous avons enregistré les verbalisations relatives aux actions et aux opérations mentales mises en œuvre dans ces situations, les actions des utilisateurs, et les dialogues impliquant le concepteur et/ou les utilisateurs. Il s'agissait de verbalisations simultanées à la réalisation de la tâche (Hoc, \& Leplat, 1983), mais également des dialogues entre les participants à propos du logiciel ou de leur activité. Les enregistrements ont été faits au moyen d'un dictaphone (audio) et du logiciel CamStudio pour les manipulations du logiciel. Les enregistrements audio qui ont été retranscrits verbatim constituaient ainsi notre corpus composé de 505 lignes.

\subsubsection{Méthode d'analyse}

30 Les données recueillies dans les deux situations d'étude ont fait l'objet de la même méthode d'analyse. La méthode a procédé en quatre étapes : segmentation du corpus en unités (une unité correspondant à chaque prise de parole d'un participant), caractérisation du participant ayant produit l'unité (statut du locuteur, i.e. concepteur ou utilisateur, et de son métier, i.e. ingénieur, designer ou marketeur), codage de chaque unité en vue de caractériser sa contribution à l'activité en situation, puis regroupement des unités en chronique d'activité. Dans la lignée du schéma de codage de Darses, Détienne, Falzon et Visser (2001) élaboré pour l'analyse et la modélisation des activités cognitives de conception collective, le contenu de chaque unité a ensuite été codé selon son type (requête ou assertion), l'activité cognitive associée (génération, évaluation positive /négative, information, validation, rejet, justification) et le sujet concerné (utilisation, spécification, solution, technique, objet). Les différents types d'activité cités s'appliquent aux tâches informatisées (par ex. le rejet peut porter sur une fonction technique) ainsi que sur d'autres dimensions de l'activité (par ex. la génération peut porter sur un nouvel usage dans leur métier). Le Tableau 1 définit chacune de ces catégories.

31 Un total de 111 unités et 132 unités a été identifié, respectivement, pour la situation de formation et pour la situation d'utilisation dans le cadre d'une revue de projet. 
Tableau 1 : Définitions et exemples de types (requête, assertion), d'activités (génération, évaluation, information, validation, rejet, justification) et de sujets (utilisation, spécification, solution, technique, objet).

Table 1: Definitions and examples of types (query, assertion), activities (generation, evaluation, information, validation, rejection, justification) and topics (use, specification, solution, technical aspects, object)

\begin{tabular}{|c|c|c|c|}
\hline & Catégorie & Définition & Exemples d'extraits \\
\hline \multirow[t]{2}{*}{ Type } & Requête & Question & Et c'est une contrainte pour toi? \\
\hline & Assertion & Affirmation & C'est juste que par contre il faudra faire 2 fichiers différents quoi. \\
\hline \multirow[t]{6}{*}{ Activité } & Génération & $\begin{array}{l}\text { Action d'exprimer de des besoins latents pouvant } \\
\text { concerner de nouvelles spécifications (par ex., } \\
\text { fonctionnalités jusqu'alors non existantes), des pistes } \\
\text { de solutions (par rapport à un problème identifié } \\
\text { intrinsèque au logiciel ou induit par ce dernier), des } \\
\text { suggestions sur l'utilisation (par ex., des conseils sur la } \\
\text { manipulation de l'outil) voire sur l'objet de la séance } \\
\text { (par ex., un concept de meuble) }\end{array}$ & $\begin{array}{l}\text { Ca sous entendrait qu'il faudrait encore d'autres personnages ([notre } \\
\text { précision : avatars supplémentaires à ajouter dans la base de données]. }\end{array}$ \\
\hline & $\begin{array}{l}\text { Evaluation } \\
+/-\end{array}$ & $\begin{array}{l}\text { Action d'évoquer des constats associés à des jugements } \\
\text { positifs ou négatifs quant à l'expérience de l'utilisateur } \\
\text { avec l'artefact }\end{array}$ & $\begin{array}{l}\text { + Vous voyez quand on met les meubles les uns à côté des autres, y a des } \\
\text { phénomènes de collision qui sont bien respectés. } \\
\text { - Mais du coup vu qu'on se trouve dans la pièce, l'ombrage a du mal à } \\
\text { suivre en fait. }\end{array}$ \\
\hline & Information & $\begin{array}{l}\text { Action d'apporter des explications ou des } \\
\text { connaissances sur un sujet donné }\end{array}$ & $\begin{array}{l}\text { Ce qui nous posait problème au départ, c'était le type de texture, parce } \\
\text { que les textures on nous avait appris par rapport à un nouveau mode de } \\
\text { rendu de faire des textures différentes, et elles passent pas avec 3D } \\
\text { Child. Par contre, en faisant avec des textures standards ça passe sans } \\
\text { problème. Et là ce que j'ai fait, c'est que j'ai construit les meubles } \\
\text { dessuite avec une texture standard. Du coup, ça passe sans problème. }\end{array}$ \\
\hline & Validation & Action d'approuver & Ouais, ça c'est un truc qu'il faudra que je règle. \\
\hline & Rejet & Action de désapprouver & C'est dur la collision. \\
\hline & Justification & Action d'argumenter & Oui parce que j'ai déplacé la pièce. \\
\hline \multirow[t]{5}{*}{ Sujet } & Utilisation & $\begin{array}{l}\text { Description de l'action réalisée avec les fonctionnalités } \\
\text { du logiciel }\end{array}$ & $\begin{array}{l}\text { Ah oui dégeler le produit, donc il se rassoit bien au sol. Alors avec la } \\
\text { souris on peut le bouger de place, clic gauche, je le pousse je le mets } \\
\text { contre le mur, on peut le lever donc là c'est avec la molette. }\end{array}$ \\
\hline & Spécification & $\begin{array}{l}\text { Citation d'un but, d'une propriété ou d'une } \\
\text { fonctionnalité du logiciel }\end{array}$ & Oui ce serait bien de la voir se pencher vers le lit et regarder son enfant. \\
\hline & Solution & $\begin{array}{l}\text { Elément de réponse à un problème identifié favorisant } \\
\text { l'appropriation du logiciel }\end{array}$ & $\begin{array}{l}\text { Ce serait stratégique d'avoir une personne à temps plein qui travaille sur } \\
\text { la mise en ambiance même pour vous pour vendre les projets et nous } \\
\text { vendre les projets au client. }\end{array}$ \\
\hline & Technique & Fonctionnement technique du logiciel & $\begin{array}{l}\text { Ça change en termes d'ombrage puisque l'ombrage joue avec un point } \\
\text { lumineux, et le point lumineux doit être vers le centre de la pièce, là où } \\
\text { se trouve la commode en ce moment. }\end{array}$ \\
\hline & Objet & Objet de la situation observée & $\begin{array}{l}\text { Les façades sont battantes en fait avec un champ soft, un décentré et du } \\
\text { coup l'enrobé est là. }\end{array}$ \\
\hline
\end{tabular}

Nous avons regroupé les unités en chroniques d'activité en vue de caractériser l'enchaînement effectif des activités cognitives et des sujets abordés. Une chronique (Figure 3) se lit de la manière suivante: dans la légende, les symboles (à gauche) représentent les activités, les initiales (à droite) représentent les sujets. La figure décrit une succession d'activités, chacune concernant un sujet (identifié par des initiales à l'intérieur des symboles).

Figure 3 : Exemple de chronique décrivant une succession d'activités réalisées par les participants impliqués dans une situation d'usage d'Appli-Viz'3D.

Figure 3 : Example of a chronicle describing a succession of activities carried out by the participants involved in a situation of use of Appli-Viz'3D

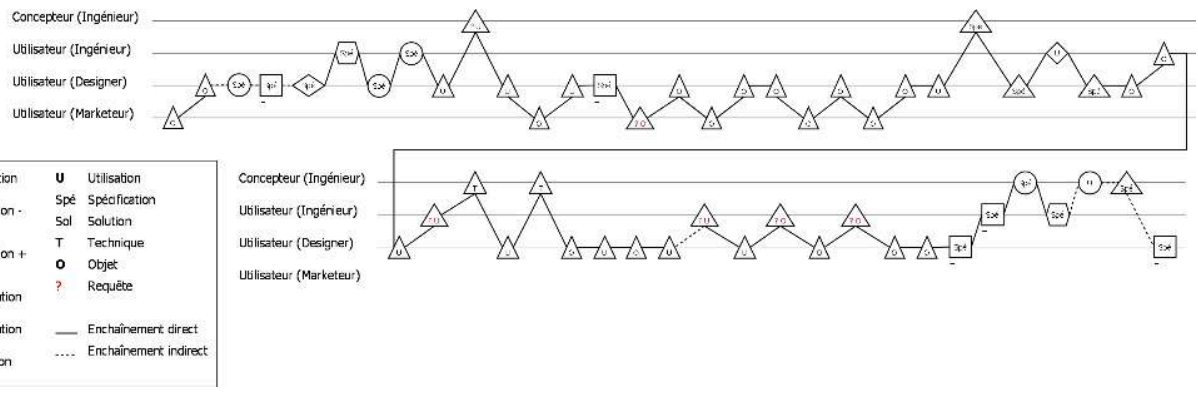

33 L'analyse statistique descriptive a porté sur les variables et les croisements entre variables. Nous avons évalué l'importance des corrélations entre variables (par ex. statut et métier du participant et activités cognitives) au moyen du V2 de $\mathrm{Cramer}^{4}$ et caractérisé le lien entre modalités particulières des variables au moyen des Taux de Liaison (TDL) 5 . 


\subsection{Résultats : situation de formation}

\subsubsection{Des contributions différentes selon les profils de participants}

L'analyse des marges effectuée à partir du Tableau 2 montre que les utilisateursdesigners sont ceux qui ont évoqué le plus d'unités (45\%), tandis que l'utilisateuringénieur est celui qui en a énoncé le moins (3\%). L'utilisateur-marketeur et le concepteur-ingénieur ont produit chacun $26 \%$ des unités. Les discussions sont composées de nombreuses assertions (91\%) et de quelques requêtes (9\%). Les deux variables «statut et métier des participants » et "type d'unité » exhibent une liaison globale forte $(\mathrm{V} 2=0,21)$. L'analyse des TDL met en évidence que le groupe des utilisateurs-marketeurs se distingue en ce qu'il est celui qui privilégie les requêtes (TDL $=+2,44$, par ex. : « et la morphologie du personnage on peut l'adapter en fait?»). Chez les concepteurs et les autres utilisateurs à l'inverse, les assertions sont privilégiées avec très peu voire aucune requête (par ex. "c'est juste que par contre il faudra faire deux fichiers différents »).

Tableau 2 : Distribution du nombre d'unités relatives à chacun des types en fonction des profils de participants, unités produites en situation de formation.

Table 2: Types vs. type of participants: number of types produced in training situation

\begin{tabular}{|l|l|l|l|l|l|}
\hline Type & Concepteur & $\begin{array}{l}\text { Utilisateur- } \\
\text { marketeur }\end{array}$ & $\begin{array}{l}\text { Utilisateur- } \\
\text { ingénieur }\end{array}$ & Utilisateur-designer & Totaux \\
\hline Requête & 1 & 9 & 0 & 0 & 10 \\
\hline Assertion & 28 & 20 & 3 & 50 & 101 \\
\hline Totaux & 29 & 29 & 3 & 50 & 111 \\
\hline
\end{tabular}

Globalement, les activités cognitives mises en œuvre sont principalement (Tableau 3) des informations ( $57 \%$ ), et dans une moindre mesure, des générations (16\%), des évaluations négatives (15\%), des justifications ( $8 \%$ ), des évaluations positives et des rejets (chacun, $2 \%$ ).

Tableau 3 : Distribution du nombre d'activités en fonction des profils de participants, activités produites en situation de formation.

Table 3: Activities vs. type of participants: number of activities produced in training situations

\begin{tabular}{|l|r|l|l|r|r|}
\hline Activité & Concepteur & $\begin{array}{l}\text { Utilisateur- } \\
\text { marketeur }\end{array}$ & $\begin{array}{l}\text { Utilisateur- } \\
\text { ingénieur }\end{array}$ & $\begin{array}{l}\text { Utilisateur- } \\
\text { designer }\end{array}$ & \multicolumn{1}{l|}{ Totaux } \\
\hline Génération & 1 & 8 & 3 & 6 & 18 \\
\hline Evaluation+ & 0 & 0 & 0 & 2 & 2 \\
\hline Evaluation- & 1 & 7 & 0 & 9 & 17 \\
\hline Validation & 0 & 0 & 0 & 0 & 0 \\
\hline Rejet & 0 & 2 & 0 & 0 & 2 \\
\hline Information & 23 & 11 & 0 & 29 & 63 \\
\hline Justification & 4 & 1 & 0 & 4 & 9 \\
\hline Totaux & 29 & 29 & 3 & 50 & 111 \\
\hline
\end{tabular}

Les variables «statut et métier des participants " et "catégorie d'activité » ont une liaison globale intermédiaire $(\mathrm{V} 2=0,12)$. L'analyse des TDL met en évidence qu'alors que le concepteur réalise des activités d'informations (TDL $=+.40$, par ex. : «si, il devrait avoir les textures, mais je pense que c'est la mémoire qui arrive à saturation») et de justifications (TDL =+.70, par ex. : [dégèle le bureau] «parce que j'ai m'impression qu'il est surélevé»), les profils d'utilisateurs déploient les autres activités de façon privilégiée 
(Figure 4) : l'évaluation positive pour les designers (TDL =+1,22, par ex. : «l'autre intérêt de ça, c'est se mettre à la place du point de vue du bonhomme»), la génération pour l'ingénieur (TDL $=+5,17$, par ex. : «il faudrait paramétrer par rapport à des côtés des dimensions, on veut le bureau à tant du mur ») et le marketeur (TDL =+.70), puis l'évaluation négative et le rejet pour le marketeur (respectivement, TDL $=+.58$ et TDL $=+2,83$, par ex. : "et là y a une fenêtre, ça va pas plus loin que ça!»).

Comme le montre le Tableau 4, les principaux sujets abordés lors de la formation concernent l'utilisation de l'artefact ( $46 \%$, par ex. : « il faut le prendre de sa base en fait » [en parlant de la sélection d'un objet 3D]) et les spécifications ( $36 \%$, par ex. : «le personnage, lui, il peut se promener dans la scène»). Les sujets les moins abordés sont les solutions (7 \%), la technique (7 \%) et l'objet (3\%).

Tableau 4 : Distribution du nombre de sujets en fonction des profils de participants, sujets produits en situation de formation.

Table 4: Subjects vs. type of participants: number of topics produced in training situations

\begin{tabular}{|l|l|l|l|l|l|}
\hline Sujet & Concepteur & $\begin{array}{l}\text { Utilisateur- } \\
\text { marketeur }\end{array}$ & $\begin{array}{l}\text { Utilisateur- } \\
\text { ingénieur }\end{array}$ & $\begin{array}{l}\text { Utilisateur- } \\
\text { designer }\end{array}$ & Totaux \\
\hline Utilisation & 18 & 8 & 0 & 25 & 51 \\
\hline Spécification & 4 & 15 & 3 & 18 & 40 \\
\hline Solution & 1 & 3 & 0 & 4 & 8 \\
\hline Technique & 5 & 1 & 0 & 2 & 8 \\
\hline Objet & 1 & 2 & 0 & 1 & 4 \\
\hline Totaux & 29 & 29 & 3 & 50 & 111 \\
\hline
\end{tabular}

Il existe une relation intermédiaire entre le «statut et métier des participants » et la «catégorie de sujets abordés » $(\mathrm{V} 2=0,07)$. Comme indiqué dans la Figure 4 , l'analyse des TDL met en évidence que le concepteur aborde des sujets relatifs à l'utilisation ( $(\mathrm{DL}=+.35)$ et à la technique ( $\mathrm{TDL}=+1,39)$, l'ingénieur traite des spécifications (TDL $=+1,78$ ), le marketeur évoque des solutions, des spécifications et des verbalisations sur l'objet de l'activité (respectivement, TDL =+.44, TDL =+.44, TDL =+.91), tandis qu'aucune attraction remarquable n'est observée entre le groupe des utilisateursdesigners et les sujets abordés.

Figure 4 : Graphe des attractions entre les profils des participants, les sujets (à gauche) et les activités (à droite) dans le cadre de la situation de formation.

Figure 4: Graph of attractions between participant profiles, subjects (left) and activities (right), in training situations

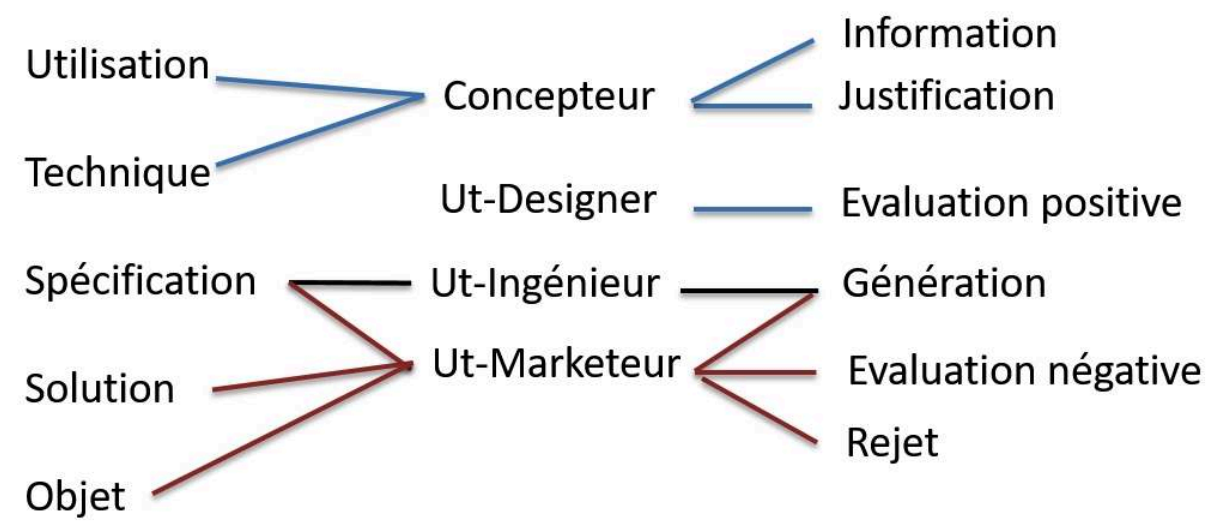




\subsubsection{Focus sur l'activité de génération}

39 concerne la proposition de nouvelles fonctionnalités et propriétés quant à l'utilisation du logiciel. Ces générations sont des assertions correspondant à 10 générations de spécifications, 6 générations de solutions et 1 génération sur l'objet. L'analyse des chroniques fait apparaitre que ces 17 générations sont précédées d'activités informationnelles (4 occurrences), évaluatives (7 occurrences), justificatives (2 occurrences), génératives (3 occurrences), et de rejet (1 occurrence). Comme le précise la Figure 5, les informations fournies préalablement aux générations concernent l'utilisation (2), l'objet (1) et la technique (1). Les évaluations portent, quant à elle, sur les spécifications ( 5 occurrences), les solutions ( 1 occurrence) et l'utilisation (1 occurrence). Les justifications font référence aux spécifications (1 occurrence) et à l'utilisation ( 1 occurrence). Les générations sont relatives aux solutions (2 occurrences) et aux spécifications (1 occurrence), et le rejet est celui d'une spécification (1 occurrence).

échanges entre les participants ont porté aussi bien sur l'amélioration d'AppliViz'3D que sur des usages potentiels. En ce qui concerne l'amélioration de l'outil, ce sont généralement les évaluations négatives de spécifications implémentées qui engendraient de nouveaux besoins en termes de fonctionnalités (par ex. «Et là y a une fenêtre, ça va pas plus loin que ça!», suivi de "Et après on pourra créer plusieurs genres de pièce? Ça peut faire partie des souhaits pour la suite des opérations si on veut pousser un peu plus loin.»). Les usages potentiels (par ex. "là où ça pourrait être un outil génial c'est par rapport à la plv») étaient évoqués suite à des informations sur l'activité (par ex. «après pour la plv en termes d'aménagement ils ont des choses importantes en 2D, mais c'est vrai qu'en $3 D$ y a des choses qui pourraient manquer »). Ces générations quant aux usages potentiels étaient souvent suivies de justifications (par ex. «Des fois quand je vois la déperdition qui est faite en magasin ou par nos commerciaux entre ce qui a été créé et ce qui est sur le terrain, comment c'est utilisé. Et là y a un vrai message qui peut être vendu avec un outil comme ça pour montrer les fonctions. Alors moi c'est le point de vue, je dirai marketing commercial») ou d'éléments de mise en œuvre dans l'activité (par ex. «Et aujourd'hui si on voulait faire ça, je dirai oui c'est vachement bien pour ça, mais il faudrait que ce soit créer entièrement par la plv à chaque fois qu'on fait un plan et aujourd'hui on est incapable [en gamme professionnelle de mobilier] de gérer la charge de travail sur un truc comme ça on a déjà du mal à avoir un plan 2D basique alors gérer un plan d'animation avec des personnages ».) 
Figure 5 : Activités (au milieu) et sujets (à droite) précédant la génération de besoins latents dans le cadre de la situation de formation.

Figure 5: Activities (middle) and subjects (right) before the generation of latent needs, in training situations

Génération

Besoins latents (17)



\subsection{Résultats : situation de revue de projet}

\subsubsection{Des contributions différentes selon les profils de participants}

L'analyse des marges effectuée à partir du Tableau 5 montre que les utilisateursdesigners sont ceux qui ont évoqué le plus d'unités (55\%), tandis que les utilisateursmarketeurs sont ceux qui en ont énoncé le moins (9\%). L'utilisateur-ingénieur et le concepteur-ingénieur ont produit respectivement $19 \%$ et $17 \%$ des unités.

Les discussions sont composées de nombreuses assertions ( $89 \%$ ) et de quelques requêtes $(11 \%)$. Les deux variables "statut et métier des participants " et "type d'unité » exhibent une liaison globale intermédiaire $(\mathrm{V} 2=0,07)$. L'analyse des TDL met en évidence que le groupe des utilisateurs-ingénieurs pose davantage de questions (TDL $=+1,46$ ), alors que les utilisateurs-designers (TDL $=-.51$ ) et les utilisateursmarketeurs (TDL $=-.27)$ privilégient plutôt les assertions.

Tableau 5 : Distribution du nombre d'unités relatives à chacun des types en fonction des profils de participants, unités produites lors de la revue de projet.

Table 5: Types vs. kind of participants: number of types produced in work situations

\begin{tabular}{|l|l|l|l|l|l|}
\hline Type & Concepteur & $\begin{array}{l}\text { Utilisateur- } \\
\text { designer }\end{array}$ & $\begin{array}{l}\text { Utilisateur- } \\
\text { ingénieur }\end{array}$ & $\begin{array}{l}\text { Utilisateur- } \\
\text { marketeur }\end{array}$ & Totaux \\
\hline Requête & 3 & 4 & 7 & 1 & 15 \\
\hline Assertion & 20 & 68 & 18 & 11 & 117 \\
\hline Totaux & 23 & 72 & 25 & 12 & 132 \\
\hline
\end{tabular}

43 L'analyse des marges effectuée à partir du Tableau 6 met en évidence que les activités cognitives mises en œuvre sont principalement des informations (69\%), et dans une moindre mesure, des évaluations négatives (14\%), des justifications $(8 \%)$, des générations (7 \%), des validations (2\%) et des évaluations positives (1\%). 
Tableau 6 : Distribution du nombre d'activités en fonction des profils de participants, activités produites lors de la revue de projet.

Table 6: Activities vs. kind of participants: number of activities produced in work situations

\begin{tabular}{|l|l|l|l|l|l|}
\hline Activité & Concepteur & $\begin{array}{l}\text { Utilisateur- } \\
\text { designer }\end{array}$ & $\begin{array}{l}\text { Utilisateur- } \\
\text { ingénieur }\end{array}$ & $\begin{array}{l}\text { Utilisateur- } \\
\text { marketeur }\end{array}$ & Totaux \\
\hline Génération & 2 & 4 & 1 & 2 & 9 \\
\hline Evaluation+ & 0 & 1 & 0 & 0 & 1 \\
\hline Evaluation- & 1 & 13 & 3 & 1 & 18 \\
\hline Validation & 0 & 0 & 2 & 0 & 2 \\
\hline Rejet & 0 & 0 & 0 & 0 & 0 \\
\hline Information & 17 & 48 & 17 & 9 & 91 \\
\hline Justification & 3 & 6 & 2 & 0 & 11 \\
\hline Totaux & 23 & 72 & 25 & 12 & 132 \\
\hline
\end{tabular}

Les variables «statut et métier des participants " et "catégorie d'activité » ont une liaison globale intermédiaire ( $\mathrm{V} 2=0,04)$. L'analyse des TDL met en évidence qu'alors que le concepteur propose plus de justifications (TDL =+.60, par ex. : «oui parce que j'ai déplacé la pièce ») et de générations (TDL =+.28), les profils d'utilisateurs se répartissent les autres activités de façon privilégiée (Figure 5 ) : les évaluations positives (TDL =+.83, par ex. : "du coup importer les meubles dans 3D Child c'est assez simple [...] faut pas très longtemps ») et négatives (TDL =+.32, par ex. : «avec les éclairages on a du mal à voir les détails de la façade en fait ») pour les designers, la validation pour l'ingénieur (TDL =+4,28, par ex. : il appuie les propos d'un designer affirmant « ben ouais parce que du coup ça nous permettrait de pivoter autour du meuble» en précisant «ce que vous n'arrivez pas à faire sur la planche ») et la génération pour les marketeurs (TDL =+1,44, par ex. : «Ce serait bien de la voir se pencher vers le lit et regarder son enfant »).

Comme le montre le Tableau 7, les principaux sujets abordés lors de la revue de projet concernent l'utilisation de l'artefact (32\%, par ex.: "Si on veut zoomer plus, il faut déplacer la pièce vers la caméra»), les spécifications (31\%, par ex. : «là ce qu'on ne sait pas, c'est l'enfant quel âge il $a$ ») et les objets (29\%, par ex. : " t'as vu sur l'extension de la table à langer, on a mis 2 traits de brulage sur les côtés là»). Les sujets les moins abordés sont la technique (5\%) et les solutions (3\%).

Tableau 7 : Distribution du nombre de sujets en fonction des profils de participants, sujets produits lors de la revue de projet.

Table 7: Subjects vs. kind of participants: number of topics produced in work situations

\begin{tabular}{|l|l|l|l|l|l|}
\hline Sujet & Concepteur & $\begin{array}{l}\text { Utilisateur- } \\
\text { designer }\end{array}$ & $\begin{array}{l}\text { Utilisateur- } \\
\text { ingénieur }\end{array}$ & $\begin{array}{l}\text { Utilisateur- } \\
\text { marketeur }\end{array}$ & Totaux \\
\hline Utilisation & 10 & 23 & 7 & 2 & 42 \\
\hline Spécification & 6 & 24 & 10 & 1 & 41 \\
\hline Solution & 1 & 2 & 1 & 0 & 4 \\
\hline Technique & 3 & 2 & 1 & 1 & 7 \\
\hline Objet & 3 & 21 & 6 & 8 & 38 \\
\hline Totaux & 23 & 72 & 25 & 12 & 132 \\
\hline
\end{tabular}

Il existe une relation intermédiaire entre le «statut et métier des participants » et la "catégorie de sujets abordés » $(\mathrm{V} 2=0,04)$. Comme indiqué dans la Figure 6 , l'analyse des TDL met en évidence que le concepteur traite essentiellement de l'utilisation, de solutions et d'aspects techniques (respectivement, $\mathrm{TDL}=+.37, \mathrm{TDL}=+.43, \mathrm{TDL}=+1,46$ ), l'ingénieur aborde les spécifications et les solutions (respectivement, TDL =+.29, TDL =+. 
32), tandis que le marketeur évoque des sujets propres à la technique et à l'objet de la revue de projet (respectivement, TDL $=+.57, \mathrm{TDL}=+1,32$ ).

Figure 6 : Graphe des attractions entre les profils des participants, les sujets (à gauche) et les activités (à droite) dans le cadre de la situation de revue de projet.

Figure 6: Graph of attractions between participant profiles, subjects (left) and activities (right), in work situations

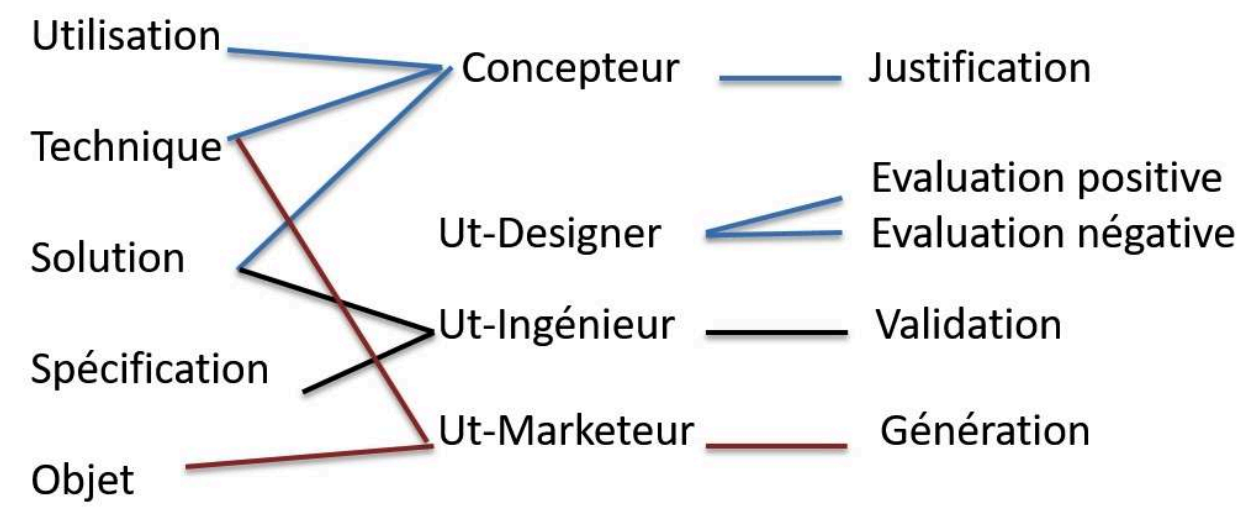

\subsubsection{Focus sur l'activité de génération}

La génération de besoins latents, représentant $7 \%$ des unités totales (c'est-à-dire, 9/132), concerne la proposition de nouvelles fonctionnalités et propriétés quant à l'utilisation du logiciel. Ces générations sont des assertions correspondant à 8 générations de spécifications et à 1 génération sur l'utilisation. L'analyse des chroniques fait apparaitre que ces 9 générations sont précédées d'activités évaluatives (1 occurrence) et informationnelles (8 occurrences). Comme le précise la Figure 7, l'évaluation porte sur l'objet de la revue de projet (c'est-à-dire, le concept de produit) et les informations fournies préalablement aux générations concernent les spécifications (3), l'utilisation (3), l'objet (1) et la technique (1).

Une analyse plus qualitative fait apparaitre que ces informations donnent lieu à trois types de besoins latents :

- Des nouvelles fonctionnalités : par ex. suite aux doutes d'un ingénieur et d'un designer sur la taille de la chambre virtuelle alors paramétrée (" elle fait pas $9 \mathrm{~m}^{2}$ ta pièce là », " elle est pas carrée»), un designer souligne une fonctionnalité manquante concernant l'absence de vérification de la taille de la pièce ;

- Le contexte d'usage : typiquement, à partir d'une information en lien avec le contenu de la revue de projet "c'est une gamme assez simple, donc c'est pas forcément là qu'on peut voir le potentiel de 3DChild", une préconisation sur l'usage futur d'Appli-Viz'3D a été énoncée: " après sur les chambres plus complexes, avec des profondeurs de gammes plus importantes, 3D Child apportera beaucoup plus ») ;

- L'insertion dans l'activité projetée : à titre d'exemple, le designer impliqué dans le projet dès son commencement expliquait la préparation des modèles 3D utilisés dans la revue de projet " ce qui nous posait problème au départ, c'était le type de texture, parce que les textures on nous avait appris par rapport à un nouveau mode de rendu de faire des textures différentes, et elles passent pas avec 3D Child. Par contre, en faisant avec des textures standards ça passe sans problème. Et là ce que j'ai fait, c'est que j'ai construit les meubles de suite avec une texture standard. Du coup ça passe sans 
problème.", ce qui a amené à une suggestion de procédure de la part d'un autre designer : "c'est juste que par contre il faudra faire deux fichiers différents ".

Figure 7 : Activités (au milieu) et sujets (à droite) précédant la génération de besoins latents dans le cadre de la revue de projet.

Figure 7: Activities (middle) and subjects (right) before the generation of latent needs, in work situations

\section{Genération \\ Besoins latents (9)}



\section{Discussion}

études présentées montrent, tout d'abord, comment l'utilité d'une technologie émergente se construit par la génération de besoins latents au travers de verbalisations et d'interactions entre un concepteur et plusieurs profils d'utilisateurs en contexte professionnel: à savoir, dans une formation et dans une situation de travail (en l'occurrence, une revue de projet de conception de mobilier de puériculture). Ainsi, les utilisateurs génèrent de nouveaux besoins lorsqu'ils sont dans des situations dans lesquelles ils utilisent le dispositif en contexte écologique, et ont accès aux informations sur les objectifs de la technologie, ses caractéristiques, son utilisation et son fonctionnement technique (au travers du concepteur présent). Ces résultats confirment les résultats d'Anastassova, Mégard et Burkhardt (2007) dans le domaine de réalité augmentée pour la formation des techniciens en maintenance automobile.

Les deux situations analysées sont clairement apparues comme complémentaires : la formation semble indispensable pour permettre aux utilisateurs de comprendre les contraintes techniques et d'usage de la technologie (typiquement, le concepteur a fourni de nombreuses informations sur la technique et l'utilisation), alors que dans la revue de projet, les utilisateurs constatent les choix de conception, justifiés par le concepteur, en produisant des activités cognitives diverses (typiquement, les utilisateurs-designers évaluaient l'artefact tandis que les utilisateurs-marketeurs s'inscrivaient dans des activités de génération). Bien que la répartition des activités cognitives et des sujets abordés différait entre les deux situations, la confrontation à la technologie a conduit les utilisateurs à générer des besoins latents et, de fait, à tendre vers une technologie plus utile pour eux.

Par ailleurs, il semblerait que la nature collective et multi-métiers des mises en situation puisse aussi avoir contribué à créer un climat propice à la créativité, comme l'ont suggéré des études antérieures (par ex. Bruseberg, \& McDonagh-Philip, 2002). En effet, nos résultats (cf. Figures 4 et 6) montrent que les participants sont complémentaires en ce qui concerne leur contribution en termes d'activités cognitives et de sujets privilégiés, et ce dans les deux situations observées. l'usage (Rabardel, \& Béguin, 2005) et montrent la nécessité de réfléchir à des stratégies 
d'introduction des technologies dans les entreprises favorisant les échanges entre concepteur et utilisateurs pour optimiser l'utilité de ces outils. En outre, le cadre méthodologique que nous avons proposé consiste à mettre en œuvre des situations favorisant les apprentissages mutuels entre concepteurs et utilisateurs lors de l'usage effectif de la technologie. Les utilisateurs découvrent la technologie de desktop-VR et apprennent à utiliser le logiciel (par ex. le concepteur explique au designer, lors de la formation, la manière dont il faut sélectionner un objet 3D en lui disant «il faut le prendre de sa base en fait " auquel un utilisateur-designer répond « de sa base d'accord, puis là si on laisse tomber il va venir sur le bureau comme ça»). Ils contextualisent son usage et en expérimentent l'apport direct dans le cadre de leur activité (par ex. un designer projette l'outil dans son activité globale «ce que je trouve intéressant par rapport à [la gamme professionnelle de mobilier], c'est pouvoir circuler au niveau de ces meubles-là et d'avoir une vision d'ensemble. À la limite, est-ce que tu as mis trop de meubles, est-ce que tu peux en rajouter, est-ce qu'il y a la place d'en mettre encore plus?»). Le concepteur appréhende l'activité des différents utilisateurs (par ex. pendant la revue de projet, un designer explique au stagiaire-concepteur et à l'utilisateur-ingénieur les raisons pour lesquelles "il faudra faire deux fichiers différents " [du produit sous 3 DS Max dont un opérationnel sous Appli-Viz'3D]. Le concepteur identifie les sources de difficultés et leurs besoins non encore implémentés/finalisés dans l'application (par ex. «Là, la maman, elle balaie la pièce, son regard de gauche à droite. Donc, on a demandé à [stagiaire-concepteur] si elle pouvait bouger la tête de haut en bas»). Ces apprentissages mutuels sont possibles à travers l'introduction dans l'entreprise d'un concepteur-stagiaire ayant un profil d'ingénieur, ce qui semble être une stratégie prometteuse. Ce dernier devient un observateur participant ayant un rôle de facilitateur dans l'accompagnement du changement en expliquant les potentialités techniques et en formant à l'utilisation. À ce propos, les résultats de nos études (cf. Figures 5 et 7 ) suggèrent que les informations et les évaluations sont les activités cognitives qui précèdent le plus souvent la génération de besoins, et ce aussi bien dans la situation de formation que dans la situation de revue de projet. Les utilisateurs semblent donc avoir besoin de ce facilitateur pour les former et les accompagner dans l'usage d'Appli-Viz'3D. Le concepteur-stagiaire a également une fonction d'acteur dans la finalisation de l'utilité de la technologie à travers la collecte de données écologiques, mais aussi le développement informatique des besoins identifiés. Cela est particulièrement important dans la mesure où la non-prise en compte des besoins par la conception est un facteur susceptible d'induire un rejet du système alors perçu comme ayant un défaut d'utilité c'est-à-dire une inadéquation entre les buts poursuivis par les utilisateurs et ceux rendus possibles par le logiciel (Loup-Escande et al., 2013).

\section{Conclusion}

Dans cet article, nous avons proposé la mise en œuvre d'une approche rétrospective selon laquelle l'évaluation de la technologie amène à la production de spécifications candidates, à travers deux situations dans lesquelles un concepteur et des utilisateurs interagissent : une formation et une revue de projet. La confrontation à la technologie a conduit les utilisateurs à générer des besoins latents et, de fait, à tendre vers une technologie plus utile pour eux. D'ailleurs, le fait que des applications actuelles (par ex. configurateurs 3D, logiciels d'aide à la conception) intègrent de façon généralisée aujourd'hui les fonctionnalités mises en évidence à l'époque suggère que le travail 
mené dans le cadre de cette étude avait effectivement permis de mettre en évidence des besoins latents, finalement avérés quelques années plus tard.

Ces besoins latents, exprimés dans la phase aval de la conception, ont pour la plupart été implémentés dans le logiciel. Ceux qui n'ont pas été implémentés ne l'ont pas été pour deux raisons. La première raison concerne la difficulté de prendre en compte des besoins non prévus à la base en cours de conception et dont le développement n'aurait pas permis de tenir les délais (par ex. "créer plusieurs structures de pièces, comme une pièce avec deux fenêtres et deux portes, en plus de celle implémentée qui comportait une fenêtre et une porte »). La deuxième raison est que le concepteur pouvait estimer qu'il avait développé les fonctionnalités nécessaires pour que les utilisateurs implémentent eux-mêmes leurs besoins (par ex. "modéliser une chambre russe »). Pour plus de détails, voir Loup-Escande, Burkhardt, Christmann, \& Richir (2014).

Ces situations sont également une stratégie d'introduction de la technologie qui s'est avérée pertinente, en ce sens que le concepteur a non seulement formé les utilisateurs à ce nouvel outil professionnel et s'est assuré à travers la revue de projet qu'ils parvenaient à l'intégrer dans leur activité de travail (Appli-Viz'3D a été utilisé comme un outil de démonstration dans des salons pour les marketeurs, et comme un moyen favorisant les échanges de points de vue lors des revues de projet pour les ingénieurs et les designers). Cette étude a montré que le stagiaire-concepteur pouvait avoir une place dans la conduite des transformations digitales en restant focalisé sur l'utilité fonctionnelle et opérationnelle du dispositif. En outre, il n'a pas eu pour rôle d'investiguer les facteurs impactant l'acceptation finale d'Appli-Viz'3D dans l'activité, ni de comprendre comment le système s'incarnait dans les pratiques professionnelles individuelles et collectives - des utilisateurs.

Malgré ces avantages, l'approche rétrospective implique un prototype hautement fidèle de la technologie et est donc adaptée pour la phase aval du processus de conception centrée sur l'utilité. Par conséquent, elle reste complémentaire, en particulier pour la phase amont de la conception, à une approche prospective qui relève de la prédiction, de la projection ou encore de l'imagination des fonctions, des propriétés et de la forme de la technologie voire de ses futurs usages. Notons toutefois que cette approche nécessite une évolution des pratiques de l'ergonome. L'ergonome spécialiste en conception de technologies émergentes s'inscrit dans le contexte de l'innovation. En ce sens, les usages visés par ces technologies ne sont pas tous connus a priori, c'est-à-dire avant que la technologie soit conçue et implémentée dans l'entreprise. L'ergonome doit alors mobiliser de nouveaux courants tels que l'ergonomie prospective qui permet d'élargir le champ des usages possibles et des besoins des utilisateurs avant que la technologie soit conçue (Brangier, \& Robert, 2014). 


\section{BIBLIOGRAPHIE}

Anastassova, M. (2006). L'analyse ergonomique des besoins en amont de la conception de technologies émergentes. Le cas de la réalité augmentée pour la formation à la maintenance automobile. Thèse de doctorat en Psychologie Ergonomique de l'Université Paris Descartes, Paris, 13 décembre 2006.

Anastassova, M., Burkhardt, J.-M., \& Mégard, C. (2007). User-Centred Design and Evaluation of Augmented Reality Systems for Industrial Applications: Some Deadlocks and Breakthroughs. In Proceedings of the $9^{\text {th }}$ Virtual Reality International Conference (VRIC), 215-224.

Anastassova, M., Mégard, C., \& Burkhardt, J.-M. (2007). Prototype Evaluation and User-Needs Analysis in the Early Design of Emerging Technologies. In J.A. Jacko (Ed), Human-Computer Interaction. Interaction Design and Usability. Lecture Notes in Computer Science, 4 550. Springer, Berlin, Heidelberg.

Bach, C., \& Scapin, D. L. (2005). Critères Ergonomiques pour les Interactions HommeEnvironnements Virtuels : définitions, justifications et exemples. Research Report 5531, INRIA.

Bastien, J. M. C., \& Scapin, D. L. (1993). Critères Ergonomiques pour l'Évaluation d'Interfaces Utilisateurs. Technical Report 156, INRIA.

Bastien, J. M. C., \& Tricot, A. (2008). L'évaluation ergonomique des documents électroniques. In A. Chevalier \& A. Tricot (Eds.), Ergonomie des documents électroniques (pp. 205-227). Paris : PUF.

Béguin, P. (2003). Design as a mutual learning process between users and designers. Interacting with Computers, 15(6), 709-730.

Blandford, A., Green, T. R. G., Furniss, D., \& Makri, S. (2008). Evaluating system utility and conceptual fit using CASSM. International Journal of Human-Computer Studies, 66(6), 393-409.

Bobillier Chaumon, M. É., Rouat, S., Laneyrie, E., \& Cuvillier, B. (2018). De l'activité DE simulation à l'activité EN simulation : simuler pour stimuler. Activités, 15(1).

Bourmaud, G. (2008). Du développement des instruments à la conception de systèmes techniques. In Proceedings of ErgoIA 2008, pp. 99-106.

Brangier, E., \& Robert, J.-M. (2014). L'ergonomie prospective : fondements et enjeux. Le Travail Humain, 77(1), 1-20.

Brooke, J. (1996). SUS: a "quick and dirty" usability scale. In P. W. Jordan, B. Thomas, B. A. Weerdmeester, \& A. L. McClelland (Eds.), Usability Evaluation in Industry (pp. 189-194). London : Taylor and Francis.

Bruseberg, A., \& McDonagh-Philip, D. (2002). Focus groups to support the industrial/product designer: a review based on current literature and designers' feedback. Applied Ergonomics, 33(1), 27-38.

Burkhardt, J.-M., \& Lubart, T. (2010). Creativity in the Age of Emerging Technology: some issues and perspectives. Creativity and Innovation Management, 19(2), 160-166.

Burkhardt, J.-M. \& Sperandio, J.-C. (2004). Ergonomie et conception informatique. In P. Falzon (Ed.), Ergonomie (pp. 437-450). Paris : PUF.

Carroll, J. M. (2000). Making use: scenarios and scenario-based design of Human-Computer Interactions. Cambridge : MIT Press. 
Darses, F., Détienne, F., Falzon, P., \& Visser, W. (2001). A method for analyzing collective design processes. Research Report RR-4258, INRIA.

D’Astous, P., Détienne, F., Visser, W. \& Robillard, P. N. (2004). Changing our view on design evaluation meetings methodology: a study of software technical evaluation meetings. Design Studies, 25, 625-655.

Darses, F., \& Wolff, M. (2006). How do designers represent to themselves the users' needs? Applied Ergonomics, 37(6), 757-764.

Davis, F. D. (1989). Perceived Usefulness, Perceived Ease of Use, and User Acceptance of Information Technology. Information Technology MIS Quarterly, 13(3), 319-340.

Dix, A., Finlay, J., Abowd, J. D., \& Beale, R. (2004). Human-Computer Interaction. 3rd edition, Pearson Education.

Durand, T., Jumel, S., \& Pons, F.-M. (2003). La promotion de l'innovation au sein des organisations. Cahiers de la recherche, $\mathrm{n}^{\circ} 2$, ISTM.

Haradji, Y., \& Faveaux, L. (2006). Évolution de notre pratique de conception (1985-2005) : modéliser pour mieux coopérer à partir des critères d'utilité, d'utilisabilité... Activités, 3(1), 1-32.

Hoc, J.-M., \& Leplat, J. (1983). Evaluation of differents modalities of verbalization in a sorting task. International Journal of Man-Machine Studies, 18, 283-306

Karsenty, L. (1991). Le dialogue de validation d'un Schéma Conceptuel de Données. Research Report $R R-1551$, INRIA.

Kjeldskov, J. (2003). Human-computer interaction design for emerging technologies: virtual reality, augmented reality and mobile computer systems. Thesis, Aalborg University, Aalborg.

Loup-Escande, E. (2011). Vers une conception centrée sur l'utilité : Une analyse de la coconstruction participative et continue des besoins dans le contexte des technologies émergentes. Résumé de thèse. Activités, 8(2). https://journals.openedition.org/activites/2637

Loup-Escande, E., Burkhardt, J.-M., Christmann, O., \& Richir, S. (2014). Needs' elaboration between users, designers and project leaders: analysis of a design process of a Virtual Realitybased software. Information and Software Technology, 56(8), 1049-1061.

Loup-Escande, E., Burkhardt, J-M, Christofol, H., \& Richir, S. (2010). 3D Child Software: a decisionmaking help tool in innovative product design. Journal of Decision System, 19(1), 9-31.

Loup-Escande, E., Burkhardt, J.-M., \& Richir, S. (2013). Anticiper et évaluer l'utilité dans la conception ergonomique des technologies émergentes : une revue. Le Travail Humain, 76(1), 25-55.

Loup-Escande, E., Dominjon, L., Perret, D., Erhel, S., Jamet, E., Michinov, N., Andriot, C., Gravez, P., \& Ragot, M. (2013, Octobre). La démarche de Conception Centrée-Utilisateur en Réalité Virtuelle : l'exemple du projet VirtualiTeach. Journées de l'Association Française de Réalité Virtuelle, Laval, France, 28-30 octobre.

Murray, H. A. (1938). Explorations in Personality. New York: Oxford University Press

Newell, A. F., Carmichael, A., Morgan, M., \& Dickinson, A. (2006). The use of theatre in requirements gathering and usability studies. Interacting with Computers, 18(5), 996-1011.

Nielsen, J. (1993). Usability Engineering. New York : Academic Press.

Nogier, J. F. (2005). Ergonomie du logiciel et design Web. Le manuel des interfaces utilisateur. Paris : Dunod. 
Rabardel, P. (1995). Les hommes et les technologies. Approche cognitive des instruments contemporains. Paris : Armand Colin.

Rabardel, P., \& Béguin, P. (2005). Instrument mediated activity: from subject development to anthropocentric design. Theoretical Issues in Ergonomic Science, 6(5), 429-461.

Robertson, S. (2001). Requirements trawling: techniques for discovering requirements. International Journal of Human-Computer Studies, 55(4), 405-421.

Schmettow, M., Bach, C., \& Scapin, D. (2014, September). Optimizing Usability Studies by Complementary Evaluation Methods. International BCS Human Computer Interaction Conference, Southport, UK, 9-12 September.

Sperandio, J.-C. (2001). Critères ergonomiques de l'assistance technologique aux opérateurs. Communication au Congrès JIM' 2001 : Interaction Homme-Machine \& Assistance, Metz, France.

Stanney, K. M., Mollaghasemi, M., Reeves, L., Breaux, R., \& Graeber, D. A. (2003). Usability engineering of virtual environments (VEs): identifying multiple criteria that drive effective $\mathrm{VE}$ system design. International Journal of Human-Computer Studies, 58(4), 447-481.

Terrade, F., Pasquier, H., Reerink-Boulanger, J., Guingouain, G., \& Somat, A. (2009). L'acceptabilité sociale : la prise en compte des déterminants sociaux dans l'analyse de l'acceptabilité des systèmes technologiques. Le Travail Humain, 72(4), 383-395.

Tromp, J. G., Steed, A., \& Wilson, J. R. (2003). Systematic Usability Evaluation and Design Issues for Collaborative Virtual Environments. Presence: Teleoperators and Virtual Environments, 12(3),

241-267.

Ulrich, T. K., \& Eppinger, S. D. (2003). Product Design and Development (3e éd.). New York : Irwin/ McGraw-Hill.

\section{NOTES}

1. Au moment de l'étude décrite dans ce papier, les applications de type desktop-VR étaient considérées comme des technologies émergentes.

2. Une application de type desktop-VR correspond à une application de Réalité Virtuelle sur écran et ordinateur de bureau.

3. Un designer, mobilisé dès le début du projet, a été impliqué dans l'étude 1 et dans l'étude 2 .

4. Compris entre 0 et 1 , la liaison est considérée forte pour V2 Cramer $>0,16$, faible pour V2 Cramer $<0,04$, et intermédiaire entre les deux.

5. Il y a attraction lorsque le TDL est positif, et répulsion lorsqu'il est négatif. Par convention, nous retiendrons comme attraction remarquable les TDL ayant une valeur absolue $\geq 0.25$.

\section{RÉSUMÉS}

Cet article vise à comprendre dans quelle mesure l'évaluation d'une technologie émergente en contexte écologique au moyen d'un prototype favorise la génération de besoins latents. Ces besoins se caractérisent par le fait qu'ils ne sont pas identifiés a priori et émergent d'interactions 
entre les participants à des situations de formation et de travail mobilisant le logiciel AppliViz'3D, au sein d'une entreprise de fabrication de meubles. Cette étude a impliqué un concepteur (stagiaire en réalité virtuelle) et sept utilisateurs ( 2 ingénieurs, 3 designers et 2 marketeurs spécialisés dans la conception de mobilier de puériculture). L'analyse des verbalisations et des interactions a mis en évidence pour les deux situations observées : 1) une complémentarité des contributions des participants en termes d'activités cognitives déployées et de sujets abordés, 2) la présence de générations de nouvelles fonctionnalités et propriétés souvent précédées d'activités d'informations et d'évaluations. En discussion, nous insistons sur la nécessité de confronter les utilisateurs à la technologie dans l'environnement réel d'utilisation non seulement pour concevoir des technologies émergentes effectivement utiles, mais aussi pour mieux les introduire dans les organisations. Nous concluons sur les apports et les limites de l'approche rétrospective pour ensuite évoquer l'approche prospective de l'utilité.

This paper aims to understand how the evaluation of an emerging technology in an ecological context using a prototype promotes the generation of latent needs arising from interactions between participants in training and work situations using the Appli-Viz '3D software, within a furniture manufacturing company. This study involved a designer (virtual reality trainee) and seven users ( 2 engineers, 3 designers and 2 marketers specialized in the design of childcare furniture). The analysis of verbalizations and interactions for the two observed situations highlighted: 1) a complementarity of the participants' contributions in terms of cognitive activities and subjects, 2) the presence of generations of new functionalities and properties often preceded by information and evaluations. In the discussion, we highlight the need to confront users with the technology in a real-world environment so as to design emerging technologies that are actually useful and to better introduce them into organizations. We conclude on the contributions and limits of the retrospective approach and then discuss the prospective approach to usefulness.

\section{INDEX}

Mots-clés : processus de conception, utilité, besoins latents, technologie émergente, interactions situées

Keywords : design process, usefulness, latent needs, emerging technologies, situated interactions

\section{AUTEURS}

\section{ÉMILIE LOUP-ESCANDE}

CRP-CPO (EA 7273), Université de Picardie Jules Verne, Chemin du Thil, 80025 Amiens, emilie.loup-escande@u-picardie.fr

\section{JEAN-MARIE BURKHARDT}

Laboratoire de Psychologie des Comportements et des mobilités, IFSTTAR, 25 allée des Marronniers, 78000 Versailles, jean-marie.burkhardt@ifsttar.fr 